\title{
Diffusion and radiation in magnetized collisionless plasmas with small-scale Whistler turbulence
}

\author{
Brett D. Keenan ${ }^{1} \dagger$ and Mikhail V. Medvedev ${ }^{1}$ \\ ${ }^{1}$ Department of Physics and Astronomy, University of Kansas, Lawrence, KS 66045, USA
}

(Received 21 December 2015; revised 4 March 2016; accepted 4 March 2016)

\begin{abstract}
Magnetized high-energy-density plasmas can often have strong electromagnetic fluctuations whose correlation scale is smaller than the electron Larmor radius. Radiation from the electrons in such plasmas - which markedly differs from both synchrotron and cyclotron radiation - is tightly related to their energy and pitch-angle diffusion. In this paper, we present a comprehensive theoretical and numerical study of particle transport in cold, 'small-scale' Whistler-mode turbulence and its relation to the spectra of radiation simultaneously produced by these particles. We emphasize that this relation is a superb diagnostic tool of laboratory, astrophysical, interplanetary and solar plasmas with a mean magnetic field and strong small-scale turbulence.
\end{abstract}

\section{Introduction}

High-energy-density plasma environments are generally the sites of turbulent, high amplitude (i.e. larger or comparable to pre-existing ambient magnetic fields) electromagnetic fluctuations, which often exist at scales below the Larmor scales. Such turbulence is a common feature of astrophysical and space plasmas, e.g. in high-Mach-number collisionless shocks and in reconnection regions in weakly magnetized plasmas (Nishikawa et al. 2003; Frederiksen et al. 2004; Spitkovsky 2008; Medvedev 2009a; Sironi \& Spitkovsky 2011, 2014; Sironi, Spitkovsky \& Arons 2013). Additionally, turbulent magnetic fields existing on 'sub-Larmor scales' play a critical role in laboratory plasmas; especially in high-intensity laser plasmas, as observed in experiments at the National Ignition Facility (NIF), OmegaEP, Hercules, Trident and others (Tatarakis et al. 2003; Ren et al. 2004; Mondal et al. 2012; Huntington et al. 2015).

Small-scale electromagnetic turbulence can be of various origin and thus have rather different properties. Weibel-like turbulence (Fried 1959; Weibel 1959; Medvedev 2009b) may occur in non-magnetized plasmas, i.e. plasmas possessing no ambient (mean) magnetic field. In contrast, several turbulence-producing electromagnetic instabilities require a pre-existing magnetic field, e.g. the Whistler-mode, mirror-mode, fire-hose, Bell's-type instability among others (Bell 1978; Lucek \& Bell 2000; Bell 2004, 2005; Gary \& Karimabadi 2006; Bykov et al. 2013; Caprioli \& Spitkovsky 2014; Lazar et al. 2014; Bai et al. 2015).

$†$ Email address for correspondence: bdkeenan@ku.edu 
If the electromagnetic fields are substantially small scale and statistically random, which is usually the case with turbulence because of the random phases of fluctuations, the paths of the particles diffusively diverge. If the turbulence is sub-Larmor scale (for the electrons) then the radiation simultaneously produced by the electrons is neither cyclotron nor synchrotron (for non-relativistic or relativistic particles, respectively) but, instead, carries information about the spectrum of turbulent fluctuations.

In our previous works, we found the relation between the transport of ultrarelativistic (Keenan \& Medvedev 2013) and non-relativistic/trans-relativistic (Keenan, Ford \& Medvedev 2015) particles in isotropic three-dimensional small-scale (mean free) magnetic turbulence and the radiation spectra simultaneously produced by these particles. In Keenan \& Medvedev (2013), we found that the radiation spectrum, in the ultrarelativistic (small deflection angle) regime, agrees with the small-angle jitter radiation prediction (Medvedev 2000, 2006; Reville \& Kirk 2010; Medvedev et al. 2011; Teraki \& Takahara 2011). Furthermore, we demonstrated that the pitch-angle diffusion coefficient is directly related to, and can readily be deduced from, the spectra of the emitted radiation. These results were then generalized to non-relativistic and trans-relativistic velocities in Keenan et al. (2015).

Our previous studies strictly considered a Weibel-like magnetic turbulence. This means that we treated the electromagnetic turbulence as static, i.e. with zero real frequency and no mean field. In this study, we will extend our model to include sub-Larmor-scale electromagnetic turbulence in plasmas with ambient magnetic fields. The instabilities, in this case, are usually driven with non-zero real frequency, and thus they induce random electric fields. For this reason, one should not only consider stochastic transport via magnetic pitch-angle diffusion, but transport via electric-field-induced energy and pitch-angle diffusion as well. Additionally, we will show that the energy diffusion coefficient is proportional to the (sub-Larmor scale) magnetic, pitch-angle diffusion coefficient. The exploitation of the inter-relation between the transport and radiative properties of these plasmas should provide a powerful diagnostic tool for examination of small-scale turbulence in magnetized plasmas.

Moreover, we omit the resonant wave-particle interactions, which support the underlying electromagnetic turbulence, from our analysis and consider non-resonant particles only; as we will show, the resonant ones constitute a nearly infinitesimal test particle population.

We will principally focus on realizations of Whistler-mode turbulence, because Whistler waves are regularly seen in a very wide variety of magnetized environments. Given certain conditions, the (temperature anisotropy) Weibel instability - in pre-magnetized plasmas - may evolve into a Whistler-mode instability (Palodhi et al. 2010); thus, for example, Whistler modes may spontaneously appear in environments where Weibel-like instabilities may take hold.

Many examples of Whistler waves in space and astrophysical plasmas exist. Whistler waves near collisionless shocks in the solar system, in particular, have been observed in situ for decades. These wave modes have additionally been strongly associated with interplanetary shocks - appearing both in the upstream and downstream regions (Fairfield 1974; Tsurutani, Smith \& Jones 1983; Ramírez Vélez et al. 2012). The solar wind turbulence also appears to host Whistler modes (Lengyel-Frey et al. 1996; Lin et al. 1998).

The rest of the paper is organized as follows. Section 2 presents the analytic theory. Sections 3 and 4 describe the numerical techniques employed and the obtained simulation results. Finally, $\S 5$ presents the conclusions. All equations appear in cgs units. 


\section{Analytic theory}

Consider a test particle (electron) moving through a non-uniform, random magnetic field with velocity, $\boldsymbol{v}$. It is a regular assumption - for example, in cosmic-ray transport theory - that the magnetic field may be decomposed as two components (Schlickeiser 1994):

$$
\boldsymbol{B}(\boldsymbol{x}, t)=\boldsymbol{B}_{0}+\delta \boldsymbol{B}(\boldsymbol{x}, t),
$$

where $\boldsymbol{B}_{0} \equiv\langle\boldsymbol{B}\rangle$ is the mean value of the field and $\delta \boldsymbol{B}(\boldsymbol{x}, t)$ is the mean-free fluctuation field, that is $\langle\delta \boldsymbol{B}\rangle=0$ but $\delta B \equiv\left\langle\delta B^{2}\right\rangle^{1 / 2} \neq 0$.

The motion of an electron in a random magnetic field is, in general, very complicated. It is the spatial scale of inhomogeneity, i.e. the correlation length of the field fluctuation that fundamentally determines the dynamics. These magnetic fluctuations are deemed sub-Larmor scale (or small scale) when the electron's fluctuation Larmor radius, $r_{L} \equiv \gamma \beta m_{e} c^{2} / e \delta B$ (where $\beta=v / c$ is the dimensionless particle velocity, $m_{e}$ is the electron mass, $c$ is the speed of light, $e$ is the electric charge and $\gamma$ is the electron's Lorentz factor) is greater than, or comparable to, the correlation length of the field, $\lambda_{B}$, i.e. $r_{L} \gtrsim \lambda_{B}$. We introduce the gyro number, which fully characterizes the small-scale regime (Keenan et al. 2015) as follows:

$$
\rho \equiv r_{L} \lambda_{B}^{-1} .
$$

Notice that we are considering only the fluctuation component of the magnetic field, $\delta \boldsymbol{B}$. This is because the motion can be separated into two components: the regular gyro motion about the mean magnetic field, and the random deflections due to the small-scale random component. In the discussion to follow, we will presuppose that $\rho \gg 1$.

Next, because the fluctuation Lorentz force on the electron is random, the electron velocity and acceleration vectors vary stochastically, leading to a random (diffusive) trajectory. Additionally, the magnetic Lorentz force acts only upon the component of velocity transverse to the local magnetic field, leading only to energy conserving (i.e. $\beta=$ constant) deflections. Only an electric field can change the particle energy. When this electric field is random, transport via energy diffusion may occur - we will explore this later.

Ignoring, for the moment, the presence of any electric fields: the electron motion has two limiting regimes - depending upon the relative strength of the magnetic fluctuations with respect to the mean field. These are a 'straight line' trajectory with random (transverse) deflections (i.e. $\delta \boldsymbol{B} \gg \boldsymbol{B}_{0}$ ), and a slightly perturbed helical motion about the mean magnetic field (i.e. $\delta \boldsymbol{B} \ll \boldsymbol{B}_{0}$ ). In the latter case, we will ignore the regular component of the motion. Doing so allows us to consider only the transport in mean free, small scale, magnetic turbulence, which we have explored previously.

This picture is, of course, only correct if any present electric fields are ignored. As we will show, the contribution to the total transport due to electric fields in small-scale Whistler turbulence is negligible.

\subsection{Transport via magnetic pitch-angle diffusion}

The pitch-angle diffusion coefficient in mean free, sub-Larmor-scale magnetic turbulence is a known function of statistical parameters. It may be obtained by considering that the electron's pitch angle experiences only a slight deflection, $\delta \alpha_{B}$, over a single magnetic correlation length. Consequently, the ratio of the change in the electron's transverse momentum, $\Delta p_{t}$, to its initial momentum, $p$, 
is $\delta \alpha_{B} \approx \Delta p_{t} / p \sim e(\delta B / c) \lambda_{B} / \gamma m_{e} v$, since $\Delta p_{t} \sim F_{L} \tau_{B}-$ where $\boldsymbol{F}_{L}=(e / c) \boldsymbol{v} \times \boldsymbol{\delta} \boldsymbol{B}$ is the transverse Lorentz force and $\tau_{B} \sim \lambda_{B} / v$ is the time to transit $\lambda_{B}$. The subsequent deflection will be in a random direction, because the field is uncorrelated over scales greater than $\lambda_{B}$. As for any diffusive process, the mean squared pitch angle grows linearly with time. Thus, the diffusion coefficient appears as Keenan \& Medvedev (2013), Keenan et al. (2015):

$$
D_{\alpha \alpha} \equiv \frac{\left\langle\alpha^{2}\right\rangle}{t}=\frac{\lambda_{B}}{\gamma^{2} c\left\langle\beta_{B}^{2}\right\rangle^{1 / 2}}\left\langle\Omega_{\delta B}^{2}\right\rangle,
$$

where $\alpha$ is the electron deflection angle (pitch angle) with respect to the electron's initial direction of motion, $\left\langle\beta_{B}^{2}\right\rangle^{1 / 2}$ is an appropriate ensemble-average over the (transverse) electron velocities, and

$$
\boldsymbol{\Omega}_{\delta B} \equiv \frac{e \delta \boldsymbol{B}}{m_{e} c} .
$$

In general, the pitch-angle diffusion will be path dependent, owing to the dependence on the magnetic correlation length, $\lambda_{B}$. To properly treat the correlation length, we must introduce the two-point autocorrelation tensor of the magnetic fluctuations (Keenan et al. 2015),

$$
R^{i j}(\boldsymbol{r}, t) \equiv\left\langle\delta B^{i}(\boldsymbol{x}, \tau) \delta B^{j}(\boldsymbol{x}+\boldsymbol{r}, \tau+t)\right\rangle_{\boldsymbol{x}, \tau},
$$

with the path and time-dependent correlation length tensor defined as:

$$
\lambda_{B}^{i j}(\hat{\boldsymbol{r}}, t) \equiv \int_{0}^{\infty} \frac{R^{i j}(\boldsymbol{r}, t)}{R^{i j}(0,0)} \mathrm{d} r .
$$

To evaluate this expression, we must consider the physics involved. In magnetic deflections, only the component of the magnetic field transverse to the particle velocity is involved in the acceleration. Thus, for magnetic fields, we only consider fields transverse to the direction of motion. In contrast, electric fields will have a longitudinal and transverse correlation length. The former is important for energy diffusion - whereas, the latter governs pitch-angle diffusion, since transverse deflections do no work.

Evaluation of (2.6) can be very difficult in a general case. If we make some simplifying assumptions about the magnetic turbulence, however, we may evaluate (2.6) exactly. If the transit time of a particle over a correlation length is shorter than the field variability time scale, then we can treat the magnetic field as static. Additionally, assuming statistical homogeneity and isotropy permits us to use a simple expression for the correlation tensor.

The pitch-angle diffusion coefficient, under these simplifying assumptions, has been derived previously (Keenan et al. 2015). We repeat those results here. The magnetic correlation length assumes the form (Keenan et al. 2015):

$$
\lambda_{B}=\frac{3 \pi}{8} \frac{\int_{0}^{\infty} k\left|\delta \boldsymbol{B}_{k}\right|^{2} \mathrm{~d} k}{\int_{0}^{\infty} k^{2}\left|\delta \boldsymbol{B}_{k}\right|^{2} \mathrm{~d} k},
$$


where $\left|\delta B_{k}\right|^{2}$ is the spectral distribution of the fluctuation magnetic field in Fourier $k$-space. Thus, the pitch-angle diffusion coefficient, for electrons moving through subLarmor-scale (isotropic/homogeneous) magnetic turbulence, is:

$$
D_{\alpha \alpha}=\frac{3 \pi}{8} \sqrt{\frac{3}{2}} \frac{\int_{0}^{\infty} k\left|\delta \boldsymbol{B}_{k}\right|^{2} \mathrm{~d} k}{\int_{0}^{\infty} k^{2}\left|\delta \boldsymbol{B}_{k}\right|^{2} \mathrm{~d} k} \frac{\left\langle\Omega_{\delta B}^{2}\right\rangle}{\gamma^{2} c \beta},
$$

where we have assumed a mono-energetic distribution of electrons with velocity, $\beta$.

In our derivation, we have considered the average square displacement in the pitch angle of an ensemble of electrons - with respect to the initial direction of motion of each representative electron. This should be contrasted with the usual convention which specifies a pitch angle with respect to the mean magnetic field; i.e. the reference direction is the same for all electrons.

However, since the assumed magnetic turbulence is statistically homogeneous and isotropic, the deflection angle, $\alpha$, for each electron may be chosen with respect to an arbitrary axis. When a mean field is present, the parallel component of the velocity is unaffected by $\boldsymbol{B}_{0}$. Consequently, without loss of generality, we can define $\alpha$ as the conventional pitch angle - i.e. the angle of the velocity vector with respect to the mean (ambient) magnetic field.

In reality, nevertheless, the turbulence will likely be anisotropic. This is certainly the case for Whistler turbulence. For this reason, our assumption of isotropy must be questioned. Nonetheless, the general expression, equation (2.3) holds for anisotropic turbulence as long as the correlation length is properly specified along the coordinate direction of interest. The regular component of the particle motion - i.e. the gyro motion about the mean magnetic field - must also be subtracted out for directions other than along $\boldsymbol{B}_{\mathbf{0}}$.

For the sake of computational and analytical simplicity, we choose to consider isotropic turbulence in this work, thereby allowing us to consider only the diffusion coefficient along the axis of the mean magnetic field - which is representative of the diffusion as a whole. We leave more realistic anisotropic cases to future studies.

\subsection{Energy diffusion in small-scale electric turbulence}

As mentioned previously, random electric fields may induce transport via energy diffusion. Although diffusive energy transport in electromagnetic turbulence has long been a topic of investigation (Stix 1992), energy diffusion in strictly sub-Larmor-scale electromagnetic fields has yet to be - to the best of our knowledge - explored. This topic has proved to be richly complicated, so we have limited ourselves to a particularly simple regime.

To begin, we must consider the time scales involved. There are two such characteristic time scales: the acceleration time, $\tau_{E}^{l}$ and the electric field autocorrelation time, $\tau_{a c}$. The latter time scale characterizes the temporal inhomogeneity of the electric field. Diffusive (energy) transport may arise, not only from spatial stochasticity in the electric field, but temporal randomness as well.

The former quantity, $\tau_{E}^{l}$, characterizes the spatial stochasticity. This is the time required to transit an electric field correlation length, $\lambda_{E}^{l}$ - with the ' $l$ ' superscript indicating the longitudinal transit time; i.e. the time required to transverse a longitudinal electric correlation length, $\lambda_{E}^{l}$, which is along the direction of motion. 
Assuming that $a_{\lambda} \tau_{E}^{l} \ll v_{E}$, where $a_{\lambda}$ is the acceleration over $\lambda_{E}^{l}$ and $v_{E}$ is the component of the electron velocity parallel to the electric field, the transit time is:

$$
\tau_{E}^{l} \sim \frac{\lambda_{E}^{l}}{v_{E}} .
$$

While transiting a single correlation length, the electron is subject to a nearly uniform electric field. These accelerations are uncorrelated on a spatial scale dictated by the electric field correlation length.

The diffusion regime we will explore will consider spatial diffusion to be the dominant process, i.e.

$$
\tau_{E}^{l} \ll \tau_{a c} .
$$

Furthermore, to ensure that the energy change is random on the time scale of consideration, we require that:

$$
\tau_{E}^{l} \ll t .
$$

Next, an equation for the electron energy, $W_{e}$, may be obtained directly from the Lorentz force equation of motion. It is:

$$
\frac{\mathrm{d} W_{e}}{\mathrm{~d} t}=e(\boldsymbol{v} \cdot \boldsymbol{E}) .
$$

Since the electron energy changes over the characteristic time scale, $\tau_{E}^{l}$, we may write:

$$
\frac{\Delta W_{\lambda}}{\tau_{E}^{l}} \sim e v_{E} E .
$$

If the random process is, indeed, diffusive:

$$
D_{W W} \equiv \frac{\left\langle W_{e}^{2}\right\rangle}{t} .
$$

Thus:

$$
D_{W W} \sim \frac{\left(\Delta W_{\lambda}\right)^{2}}{\tau_{E}^{l}} \sim e^{2} v_{E} E^{2} \lambda_{E}^{l},
$$

where we have used (2.9). With the usual assumptions of statistical homogeneity/ isotropy and an initially mono-energetic distribution of electrons, we may write the energy diffusion coefficient, thus:

$$
D_{W W}=\sqrt{\frac{1}{3}} e^{2}\left\langle E^{2}\right\rangle v \lambda_{E}^{l} .
$$

This result may be contrasted with the temporal, i.e. resonant, energy diffusion coefficient. The physics of this type of diffusion may be understood by considering the so-called quasilinear energy diffusion coefficient. Typically, a velocity space or momentum space diffusion coefficient is desired in the quasilinear regime - since these are connected to the transport coefficients in the Fokker-Planck equation (Schlickeiser 1994; Giacalone \& Jokipii 1999). In our case however, the energy diffusion coefficient is of greater interest, since it is directly proportional to the electric correlation length - a key turbulent statistical parameter.

Nevertheless, the physical intuition behind the quasilinear energy and velocity/ momentum diffusion coefficients is the same. Following Stix (1992), we will consider 
only small corrections to the electron's initial velocity - hence, we will assume the zero-order trajectory:

$$
\boldsymbol{r}(t)=\boldsymbol{v} t+\boldsymbol{r}_{0},
$$

where $\boldsymbol{r}_{0}$ is the electron's initial position. Let us suppose that the electric field assumes a simple sinusoidal profile, i.e.

$$
\boldsymbol{E}(\boldsymbol{x}, t)=\boldsymbol{E}_{0} \cos (\boldsymbol{k} \cdot \boldsymbol{x}-\Omega t) .
$$

Thus, using (2.12) and (2.18), we have:

$$
\frac{\mathrm{d} W_{e}}{\mathrm{~d} t}=e\left(\boldsymbol{v} \cdot \boldsymbol{E}_{0}\right) \cos \left(\boldsymbol{k} \cdot \boldsymbol{v} t+\boldsymbol{k} \cdot \boldsymbol{r}_{0}-\Omega t\right) .
$$

Integrating (2.19), averaging over all possible initial positions and squaring the result, gives the energy variance:

$$
\left\langle\Delta W_{e}^{2}\right\rangle=\left[\frac{e\left(\boldsymbol{v} \cdot \boldsymbol{E}_{0}\right)}{(\Omega-\boldsymbol{k} \cdot \boldsymbol{v})}\right]^{2} \sin ^{2}\left[\frac{(\Omega-\boldsymbol{k} \cdot \boldsymbol{v}) t}{2}\right] .
$$

Finally, with $\Omega t \gg 1$, we may employ the relation (Stix 1992):

$$
\sin ^{2}\left[\frac{(\Omega-\boldsymbol{k} \cdot \boldsymbol{v}) t}{2}\right] \sim \pi \delta(\Omega-\boldsymbol{k} \cdot \boldsymbol{v}),
$$

Thus the (quasilinear) diffusion coefficient is:

$$
D_{W W}^{r e s .} \equiv \frac{\left\langle\Delta W_{e}{ }^{2}\right\rangle}{t} \sim \pi\left[\frac{e\left(\boldsymbol{v} \cdot \boldsymbol{E}_{0}\right)}{(\Omega-\boldsymbol{k} \cdot \boldsymbol{v})}\right]^{2} \delta(\Omega-\boldsymbol{k} \cdot \boldsymbol{v}) .
$$

In general, turbulence will contain a spectrum of waves; hence, an integration of (2.22) over $\left|\boldsymbol{E}_{k, \Omega}\right|^{2}$ is required to produce the complete diffusion equation.

Nevertheless, much can be gathered by examining the functional form of this simplified expression. For example, owing to the $\delta(\Omega-\boldsymbol{k} \cdot \boldsymbol{v})$ dependence, only particles that are in resonance with the wave participate in the diffusive process.

Significantly, the quasilinear diffusion equation derived here applies to nonmagnetized plasmas. When an ambient magnetic field, $\boldsymbol{B}_{0}$, is present, the resonance condition generalizes to Stix (1992) and Schlickeiser (1994):

$$
\Omega-k_{\|} v_{\|}=n \Omega_{c e} / \gamma
$$

where $\Omega_{c e} \equiv e B_{0} / m_{e} c$ is the non-relativistic gyro frequency, the parallel direction is along the ambient (mean) magnetic field and $n$ is an integer. Typically, only the $n=$ $0, \pm 1, \pm 2$ resonances need to be considered for Whistler waves; however, sufficiently high-energy electrons may have access to the higher-order resonances (Chang et al. 2014).

Equation (2.23) may, alternatively, be expressed as:

$$
\frac{\Omega}{k v}-\cos \left(\theta_{k}\right) \cos (\alpha)=\frac{n}{k r_{L 0}^{r e l .}},
$$


where $r_{L 0}^{r e l .}=\gamma r_{L 0}, r_{L 0}=e B_{0} / m_{e} c$ is the non-relativistic Larmor radius in the mean magnetic field, $\theta_{k}$ is the angle between $\boldsymbol{k}$ and $\boldsymbol{B}_{\mathbf{0}}$ and $\alpha$ is the conventional pitch angle. If we suppose that the magnetic field is small scale, i.e. $k r_{L 0}^{r e l .} \gg 1$, then we have:

$$
\frac{\Omega}{k v}-\cos \left(\theta_{k}\right) \cos (\alpha) \simeq 0 .
$$

The implication of (2.25) is that cyclotron resonances (i.e. $|n|>0$ ) are not important for electrons moving through sub-Larmor-scale fields. However, we have, thus far, not ruled out the Landau resonant interaction (i.e. $n=0$ ).

To proceed, we require a dispersion relation, $\Omega_{r}(\boldsymbol{k})$. For cold Whistler waves with wavelengths much greater than the electron skin depth, $\Omega_{r}=k^{2} c^{2} \Omega_{c e} / \omega_{p e}^{2} \cos \left(\theta_{k}\right)-$ where $\omega_{p e}$ is the electron plasma frequency. Thus, we may write:

$$
\left(\frac{k c}{\omega_{p e}}\right)\left(\frac{\Omega_{c e}}{\omega_{p e}}\right) \simeq \beta_{\|} .
$$

However, $\omega_{p e} \gg \Omega_{c e}$ and $k c \ll \omega_{p e}$ in the regime of interest. Therefore, $\beta_{\|} \sim 0$. This means that the Landau resonance $(n=0)$ for these test electrons favours the central part of the test particle distribution function, $f_{e}$; i.e. near $\beta_{\|} \sim 0$, which implies that $\partial f_{e} / \partial v_{\|} \sim 0$. Thus, these electrons do not appreciatively contribute to the growth or damping of the electromagnetic fluctuations, and thus their energy exchange with the waves is minimal.

Moreover, since $\Omega t \gg 1$, this quasilinear diffusion via a Landau resonance occurs on a much greater time scale than $\tau_{E}^{l}$. For this reason, the non-resonant energy diffusion coefficient $-(2.15)$ - is much greater than the Landau equivalent; i.e. (2.22).

\subsection{Particle transport in magnetized plasmas with electric fluctuations}

As mentioned previously, the combined effect of electric and magnetic fields can lead to fairly complicated particle dynamics. Particle drifts, for example, involving both the electric and magnetic fields, should be considered. Here, we present two realizations of the drift phenomenon. We will subsequently argue that these effects are of negligible importance for diffusion in small-scale fields.

In $\S 2.1$, we argued that sub-Larmor-scale magnetic fluctuations result in trajectories that occupy the small deflection angle regime. For this reason, the 'guiding centre approximation', that underlies the drift theory, breaks down. Consequently, the notions of curvature drift and Grad-B drift lose all meaning in this regime.

Nonetheless, a magnetized plasma contains a large-scale magnetic field - which is, by construction, 'super-Larmor scale'. Hence, drifts that involve the electric field and the ambient (mean) field are, in principle, important to consider.

The first of these that we will explore is the, so called, E cross B drift. We will, once more, assume a sinusoidal electric field. In this case, however, we assume that an ambient magnetic field, $\boldsymbol{B}_{0}$, is present. Furthermore, we suppress the time dependence. The solution for an arbitrary electric field is well known, provided that $k r_{L 0} \ll 1$. The constant drift velocity, in this case, is Chen (1984):

$$
\boldsymbol{v}_{\boldsymbol{E} \times \boldsymbol{B}}=c\left(1+\frac{1}{4} r_{L 0}^{2} \nabla^{2}\right) \frac{\boldsymbol{E} \times \boldsymbol{B}_{0}}{B_{0}^{2}} .
$$

The second term, i.e. that which involves the Laplacian operator, is a correction known as the finite-Larmor-radius effect. When $k r_{L 0} \gg 1$, the Larmor radius is much 
larger than the field wavelength. In this case, the particle is acted upon by the electric field on a time scale much shorter than the gyro period. Consequently, the drift approximation is not appropriate for small-scale fields, since the perturbation is implicitly assumed to act on a time scale of many gyro periods.

A similar drift phenomenon occurs when we consider the time dependence of the electric field. Assuming that $\Omega^{2} \ll \Omega_{c e}^{2}$, the particle will drift with velocity (Chen 1984):

$$
\boldsymbol{v}_{p}= \pm \frac{c}{\Omega_{c e} B_{0}} \frac{\mathrm{d} \boldsymbol{E}}{\mathrm{d} t} .
$$

The quantity, $\boldsymbol{v}_{p}$, is known as the polarization drift velocity. Similarly, the small-scale processes - by construction - occur on time scales much shorter than $\Omega^{-1}$. Hence, the polarization drift time scale will be far greater than $\tau_{E}^{l}$. For this reason, polarization drift is not significant on the time scales of immediate interest.

In the next subsection, we will consider the case of small-scale energy diffusion in isotropic, small-scale Whistler turbulence.

\subsection{Energy diffusion in small-scale Whistler turbulence}

Next, to evaluate (2.16), we consider a concrete example of electromagnetic turbulence in a magnetized plasma. Whistler-mode turbulence in a cold plasma admits a simple dispersion relation (Sazhin 1993):

$$
\Omega_{r}(k)=\Omega_{c e} \frac{k^{2} c^{2}}{k^{2} c^{2}+\omega_{p e}^{2}} \cos \left(\theta_{k}\right),
$$

where $\theta_{k} \in(0, \pi / 2)$. We will assume a (nearly) steady state, so that the instability is nonlinearly saturated; that is the instability growth rate, $\Omega_{i}$, is much less than all relevant frequency scales, and thus it is negligible. This treatment assumes that the turbulence is linear, i.e. $\delta B \ll B_{0}$. We will further assume that:

$$
\frac{\gamma v}{\Omega_{c e}}>\lambda_{B}
$$

where $\Omega_{c e} / \gamma$ is the relativistic gyro frequency.

Equation (2.30) implies that $\rho \gg 1$, since $\delta B \ll B_{0}$ - thus, the test electrons are sub-Larmor scale with respect to the fluctuation magnetic field, $\delta \boldsymbol{B}$.

It is worth mentioning that, formally, the cold plasma approximation requires that $k v / \Omega_{c e} \ll 1$ (Verkhoglyadova, Tsurutani \& Lakhina 2010). This condition would imply that the electron population is super-Larmor scale with respect to the magnetic field, since $\lambda_{B} \sim k_{B}^{-1}$, where $k_{B}$ is the wavenumber of the dominant wave mode. For this reason, our model implicitly presupposes the existence of a cold population of super-Larmor-scale electrons which support the Whistler modes. Consequently, our test particles will be comprised of a hot, albeit smaller, population of sub-Larmor-scale electrons. This situation may be approximately realized by the super-halo electron population (Lin 1998; Wang et al. 2015), as it propagates through the colder solar wind turbulence, which appears to include small-scale kinetic-Alfvén and Whistler-wave modes (Che, Goldstein \& Viñas 2014).

An examination of (2.29) reveals that $\Omega_{r}(k) \ll \Omega_{c e}$ in the regime where $k c \ll \omega_{p e}$. Restricting ourselves to this regime motivates the introduction of a new parameter, which we call the skin number. It is:

$$
\chi \equiv d_{e} \lambda_{B}^{-1}
$$


where $d_{e} \equiv c / \omega_{p e}$, is the electron skin depth. Thus, the regime of interest is characterized by $\chi \ll 1$.

It is noteworthy that, in principle, the test electron velocities may be large enough so that $\Omega_{c e} / \gamma \sim \Omega_{r}$. By restricting the electron velocities to the mildly relativistic regime, we may safely presuppose that the field-variability time, $\Omega_{r}^{-1}$, is sufficiently greater than the time to transit a magnetic correlation length, thus permitting the static field treatment for the magnetic field and avoiding the wave-particle resonance treatment.

Next, in the $\chi \ll 1$ regime, the electric field perpendicular to $\boldsymbol{B}_{0}$ is much greater than the component parallel to the ambient magnetic field; i.e. $E_{\perp} \gg E_{\|}$(Sazhin 1993). Furthermore, it can be shown that in the frame moving along the direction of $\boldsymbol{B}_{0}$ with velocity equal to the parallel phase velocity, $v_{p h}^{\|} \equiv \Omega_{r} / k_{\|}$, the perpendicular electric field is approximately zero (Sazhin 1993). Consequently, this allows us, via Lorentz transformation of the electromagnetic fields, to relate the magnetic spectral distribution to the electric distribution. It is, thus:

$$
\left|\boldsymbol{E}_{\boldsymbol{k}}\right|^{2} \approx\left|\boldsymbol{E}_{\boldsymbol{k}}^{\perp}\right|^{2} \approx \beta_{p h}^{2}\left|\delta \boldsymbol{B}_{\boldsymbol{k}}^{\perp}\right|^{2},
$$

where $\perp$ refers to the spectrum perpendicular to the mean magnetic field, and

$$
\beta_{p h} \equiv \frac{v_{p h}^{\|}}{c}=\frac{\Omega_{r}(\boldsymbol{k})}{k_{\|} c}
$$

Given isotropic/homogeneous magnetic turbulence:

$$
\left|\delta \boldsymbol{B}_{k}^{\perp}\right|^{2}=\left|\delta \boldsymbol{B}_{k}\right|^{2} \cos ^{2}\left(\theta_{k}\right) .
$$

This relation then allows us to express $\left\langle E^{2}\right\rangle$ in terms of the magnetic field as:

$$
\left\langle E^{2}\right\rangle=\frac{2}{3} \frac{\int \beta_{p h}^{2}\left|\delta \boldsymbol{B}_{k}\right|^{2} \mathrm{~d} \boldsymbol{k}}{\int\left|\delta \boldsymbol{B}_{k}\right|^{2} \mathrm{~d} \boldsymbol{k}}\left\langle\delta B^{2}\right\rangle .
$$

Next, the electric field correlation length may be obtained from the electric field correlation tensor. For isotropic turbulence, one may write the general expression for the Fourier image of the electric field two-point autocorrelation tensor as:

$$
\Phi_{i j}(\boldsymbol{k})=\left|\boldsymbol{E}_{k}^{t}\right|^{2}\left(\delta_{i j}-\frac{k_{i} k_{j}}{k^{2}}\right)+\left|\boldsymbol{E}_{k}^{l}\right|^{2} \frac{k_{i} k_{j}}{k^{2}} .
$$

Isotropy is an approximation here, given the polar asymmetry indicated by (2.34). Using Maxwell's equations, we may relate the longitudinal, $\left|\boldsymbol{E}_{\boldsymbol{k}}^{l}\right|^{2}$ and transverse, $\left|\boldsymbol{E}_{\boldsymbol{k}}^{t}\right|^{2}$ distributions to $\left|\delta \boldsymbol{B}_{k}\right|^{2}$ (where longitudinal and transverse are with respect to the wave vector, not the electron velocity). To wit:

$$
\left.\begin{array}{l}
\left|\boldsymbol{E}_{\boldsymbol{k}}^{t}\right|^{2}=\frac{\Omega_{r}^{2}}{k^{2} c^{2}}\left|\delta \boldsymbol{B}_{k}\right|^{2} \\
\left|\boldsymbol{E}_{\boldsymbol{k}}^{l}\right|^{2}=\left|\boldsymbol{E}_{\boldsymbol{k}}\right|^{2}-\left|\boldsymbol{E}_{\boldsymbol{k}}^{t}\right|^{2} .
\end{array}\right\}
$$


In the $\chi \ll 1$ regime, we may substitute (2.32) to express the tensor completely in terms of the magnetic spectrum. The trace of the correlation tensor is then given by:

$$
\operatorname{Tr}[\stackrel{\leftrightarrow}{\Phi}(\boldsymbol{k})]=2 \beta_{p h}^{2}\left|\delta \boldsymbol{B}_{k}\right|^{2} \cos ^{2}\left(\theta_{k}\right) .
$$

While integrating (2.38) along a selected path, we only consider the component of the electric field parallel to the trajectory, owing to the dot product with velocity in (2.12). This allows us to draw an analogy to the 'mono-polar' (magnetic) correlation length considered in Keenan et al. (2015) - permitting us to write the expression immediately as:

$$
\lambda_{E}^{l} \equiv \lambda_{E}^{T r}(x \hat{x})=\frac{3 \pi}{4} \frac{\int\left(v_{p h}^{\|}\right)^{2} k\left|\delta \boldsymbol{B}_{k}\right|^{2} \mathrm{~d} k}{\int\left(v_{p h}^{\|}\right)^{2} k^{2}\left|\delta \boldsymbol{B}_{k}\right|^{2} \mathrm{~d} k},
$$

where the integration path was chosen to be along the $x$-axis. By comparing (2.7) to (2.39), we see that the electric correlation length differs from the magnetic correlation length only by a factor of a few. For this reason, we may conclude that $\tau_{F}^{l}$ is less than $\tau_{a c} \sim \Omega_{r}^{-1}$. Consequently, the energy diffusion will be dominated by the electric field's spatial stochasticity, as per (2.10).

Additionally, $\chi \ll 1$ and $\Omega_{c e} \ll \omega_{p e}$ demand that $v_{p h}^{\|} \ll c$. This implies that $\left\langle\delta B^{2}\right\rangle \gg\left\langle E^{2}\right\rangle$. Consequently, the pitch-angle diffusion will be dominated by the magnetic deflections, and thus we may neglect the contribution due to the electric field.

Finally, given (2.35), the energy diffusion coefficient may be related directly to the (magnetic) pitch-angle diffusion coefficient via the relation:

$$
D_{W W}=\frac{2 \sqrt{2}}{9} W_{e}^{2} \beta^{2} \frac{\int\left(\beta_{p h}^{\|}\right)^{2}\left|\delta \boldsymbol{B}_{k}\right|^{2} \mathrm{~d} \boldsymbol{k}}{\int\left|\delta \boldsymbol{B}_{k}\right|^{2} \mathrm{~d} \boldsymbol{k}} \frac{\lambda_{E}^{l}}{\lambda_{B}} D_{\alpha \alpha} .
$$

Equations (2.40) and (2.8) will be confirmed, given isotropic small-scale Whistler turbulence, via first-principle numerical simulation in $\S 4$.

\subsection{Radiation production in magnetized plasmas with sub-Larmor-scale magnetic fluctuations}

As mentioned previously, radiation production by electrons moving through magnetic turbulence has been explored thoroughly by a number of authors. Typically, radiation from particles in small-scale magnetic fields will markedly differ from synchrotron and cyclotron radiation. This is because the synchrotron/cyclotron prescription requires that the magnetic field be only weakly inhomogeneous on typical Larmor spatial scales which may not hold on kinetic scales, as is the case with magnetic fields driven by Weibel-like instabilities.

For example, in the nonlinear evolution of the Weibel instability, a soliton magnetic wave may take hold. Electrons moving through this wave will emit radiation, which owing to the small-scale nature of the field, will not be of the synchrotron/cyclotron type (Schaefer-Rolffs, Lerche \& Tautz 2009; Tautz \& Lerche 2012).

Regardless of the nature of the magnetic field, the ultrarelativistic regime, specifically, is characterized by a single parameter, the ratio of the deflection angle, 
$\delta \alpha_{B}$ (over a single magnetic correlation length) to the relativistic beaming angle, $\Delta \theta \sim 1 / \gamma$. The ratio (Medvedev 2000; Medvedev et al. 2011; Keenan \& Medvedev 2013):

$$
\frac{\delta \alpha_{B}}{\Delta \theta} \sim \frac{e \delta B}{m_{e} c^{2}} \lambda_{B} \equiv \delta_{j},
$$

is known as the jitter parameter. If $\delta_{j} \ll 1$, which implies that $\rho \gg 1$, then a distant observer in the line-of-sight will see the radiation along virtually the entire trajectory of the particle. This is why the radiation from a Weibel soliton wave is not synchrotron radiation. Similarly, if the magnetic field is turbulent on these small scales, the radiation will be further distinguished; it is known as small-angle jitter radiation (Medvedev 2000, 2006; Medvedev et al. 2011). The jitter radiation spectrum is wholly determined by $\delta_{j}$ and the magnetic spectral distribution. Consider an isotropic power-law magnetic spectrum for a time-independent field, such as:

$$
\left|\boldsymbol{B}_{k}\right|^{2}= \begin{cases}C k^{-\mu}, & k_{\min } \leqslant k \leqslant k_{\max } \\ 0, & \text { otherwise, }\end{cases}
$$

where the magnetic spectral index, $\mu$, is a real number, and $C$ is a normalization. It has been shown Medvedev (2006), Reville \& Kirk (2010), Medvedev et al. (2011) and Teraki \& Takahara (2011) that mono-energetic ultrarelativistic electrons in this prescribed sub-Larmor-scale turbulence produce a flat angle-averaged spectrum below the spectral break and a power-law spectrum above the break, that is:

$$
P(\omega) \propto \begin{cases}\omega^{0}, & \text { if } \omega<\omega_{j}, \\ \omega^{-\mu+2}, & \text { if } \omega_{j}<\omega<\omega_{b}, \\ 0, & \text { if } \omega_{b}<\omega\end{cases}
$$

where the spectral break is

$$
\omega_{j}=\gamma^{2} k_{\min } c
$$

which is called the jitter frequency. Similarly, the high-frequency break is

$$
\omega_{b}=\gamma^{2} k_{\max } c .
$$

Recently, we have generalized the small-scale jitter regime to non-relativistic and mildly relativistic velocities (Keenan et al. 2015). Non-relativistic jitter radiation, or pseudo-cyclotron radiation, differs markedly from both synchrotron and cyclotron radiation. Since relativistic beaming is not realized in the non-relativistic regime, the jitter parameter loses its meaning here. Instead, the gyro number characterizes the regime, i.e. $\rho \gg 1$. Given a mono-energetic distribution of electrons, and the spectral distribution indicated by (2.42), the pseudo-cyclotron spectrum has a slightly more complicated structure than ultrarelativistic jitter radiation. It appears as Keenan et al. (2015):

$$
P(\omega) \propto \begin{cases}A+D \omega^{2}, & \text { if } \omega \leqslant \omega_{j n} \\ F \omega^{-\mu+2}+G \omega^{2}+K, & \text { if } \leqslant \omega_{b n} \\ 0, & \text { if } \omega>\omega_{b n},\end{cases}
$$

where $\mu \neq 2$ and $A, D, F, G$ and $K$ are functions of spectral/particle parameters (e.g. $\mu, k_{\min }$ and $\beta$ ). The break frequencies generalize to non-relativistic velocities as expected, namely:

$$
\omega_{j n}=k_{\min } \beta c,
$$


and the break frequency indicated by the smallest spatial scale, i.e. the maximum wavenumber, becomes:

$$
\omega_{b n}=k_{\max } \beta c .
$$

Finally, a series of Lorentz transformations allow the generalization of these results to all velocities (Keenan et al. 2015).

The introduction of a mean magnetic field will complicate this picture. The topic of radiation production by ultrarelativistic electrons in magnetized plasmas with smallscale magnetic fluctuations was originally considered in Toptygin \& Fleishman (1987). In the case of strictly sub-Larmor-scale magnetic turbulence, with a mean field, the spectrum will be the sum of a synchrotron/cyclotron component (corresponding to the mean magnetic field) and the jitter contribution from the small-scale fluctuations, i.e.

$$
P(\omega)=P_{\text {jitter }}(\omega)+P_{\text {synch }}(\omega) .
$$

Since a plasma is a dielectric medium, dispersion may affect the form of the radiation spectrum. The effect is mostly negligible in the ultrarelativistic limit, but dispersion may be required for a complete description of the mildly relativistic and non-relativistic regimes - in real plasmas. Nonetheless, the dispersion-corrected spectrum has already been considered for small-angle jitter radiation, (Keenan et al. 2015) and synchrotron radiation (Zheleznyakov \& Trakhtengerts 1966). For this reason, we will ignore plasma dispersion in this study.

Furthermore, since $\left\langle E^{2}\right\rangle \ll\left\langle\delta B^{2}\right\rangle$ for small-scale Whistler turbulence, we can completely ignore this electric contribution.

In $\S 4$, we will confirm (2.49) (via ab initio numerical simulation) in the case of small-scale (isotropic) Whistler turbulence.

\section{Numerical model}

In $\S 2$, we made a number of theoretical predictions concerning the transport and radiation properties of magnetized plasmas with small-scale turbulent electromagnetic fluctuations. Additionally, we considered a concrete realization of this in the form of a cold, magnetized plasma embedded in sub-Larmor-scale Whistler turbulence. Here we describe the first-principle numerical simulations we employed to test our predictions.

As stated previously, we assumed the existence of a background of cold plasma which supports Whistler-mode turbulence. We then inject a smaller population of hot electrons (test particles) that are sub-Larmor scale with respect to these preset Whistler magnetic fields. First, we consider the numerical generation of the Whistler magnetic and electric fields.

Our principal assumption, in generating electromagnetic turbulence, is that these stochastic electromagnetic fields are the linear superposition of a large number of wave modes with randomized propagation direction and relative phase. From this, we may construct the turbulent fields directly from the plasma waves which are characteristic of the underlying instability. This is a typical assumption used in cosmic-ray transport theory, and it holds well in the context of space/interstellar plasmas (Schlickeiser 1994; Giacalone \& Jokipii 1999; Tautz \& Dosch 2013).

In general, the properties of these electromagnetic wave modes, and their dispersion relation, are derived from the plasma dielectric tensor - the determinant, of which, provides a system of characteristic equations. Given the cold plasma approximation, these equations admit the dispersion relation specified by (2.29) - valid in the frequency range (Verkhoglyadova et al. 2010):

$$
\Omega_{c i} \ll \Omega_{r} \ll \Omega_{c e}
$$


where $\Omega_{c i} \equiv e B_{0} / m_{i} c$ is the ion cyclotron frequency and $m_{i}$ is an ion mass. The inequality is understood to hold for all ion species. The equations, additionally, specify the polarization of the wave modes. Given obliquely (with respect to the ambient magnetic field) propagating Whistler waves, the magnetic component will be right circularly polarized with the following relations among its components (Sazhin 1993; Verkhoglyadova et al. 2010)

$$
\delta B_{x}=-\frac{1}{\tan \left(\theta_{k}\right)} \delta B_{z}=\mathrm{i} \cos \left(\theta_{k}\right) \delta B_{y},
$$

where $\boldsymbol{B}_{0}$ is along the $z$-direction, and the wave vector is in the $x-z$ plane. Because the magnetic field is divergenceless, $\boldsymbol{k} \perp \delta \boldsymbol{B}$. Given these conditions, the magnetic field will rotate about the direction of the wave vector - which, in the $\chi \ll 1$ regime, will have a period much greater than all other relevant time scales. Since the phase is randomized for each wave mode, this indicates that the magnetic field is approximately linearly polarized with a random polarization axis.

Next, the electric field is (generally) elliptically polarized. It obeys the following relations (Sazhin 1993; Verkhoglyadova et al. 2010):

$$
\left.\begin{array}{l}
E_{x} / E_{y}=-\mathrm{i} \Theta_{1} \\
E_{z} / E_{x}=\Theta_{2},
\end{array}\right\}
$$

where

$$
\left.\begin{array}{l}
\Theta_{1} \equiv \frac{k^{2} c^{2} \sin \left(\theta_{k}\right) \cos \left(\theta_{k}\right)}{\omega_{p e}^{2}+k^{2} c^{2} \sin ^{2}\left(\theta_{k}\right)} \\
\Theta_{2} \equiv \frac{\Omega_{r}^{2} \omega_{p e}^{2}+\left(\Omega_{r}^{2}-\Omega_{c e}^{2}\right) k^{2} c^{2}}{\Omega_{r} \omega_{p e}^{2} \Omega_{c e}} .
\end{array}\right\}
$$

These equations suggest that the electric field parallel to the ambient magnetic field may be expressed in terms of the magnetic fluctuations via the relation (Sazhin 1993):

$$
\left|\boldsymbol{E}_{\boldsymbol{k}}^{z}\right|=\frac{\Omega_{r}^{2}}{\Omega_{c e} k c}\left|\boldsymbol{B}_{\boldsymbol{k}}\right| \tan \left(\theta_{k}\right) .
$$

Then, specifying a magnetic spectral distribution, e.g. (2.42), allows the complete description of each wave mode. We then add a large number of these waves (given random relative phases and $k$-vectors) to simulate Whistler turbulence.

Next, we describe the numerical solution of the equation of motion for our test electrons. Obviously, the test particles do not interact with each other, nor do they induce any fields. Additionally, any radiative energy losses are neglected. An individual electron's motion is, consequently, determined only by the Lorentz force equation given by:

$$
\frac{\mathrm{d} \boldsymbol{\beta}}{\mathrm{d} t}=-\frac{1}{\gamma}\left[\boldsymbol{\Omega}_{E}+\boldsymbol{\beta} \times \boldsymbol{\Omega}_{B}-\boldsymbol{\beta}\left(\boldsymbol{\beta} \cdot \boldsymbol{\Omega}_{E}\right)\right],
$$

where $\boldsymbol{\Omega}_{E} \equiv e \boldsymbol{E} / m_{e} c$ and $\boldsymbol{\Omega}_{B} \equiv \Omega_{c e} \hat{z}+\boldsymbol{\Omega}_{\delta B}$.

Equation (3.6) was solved via a fixed step fourth-order Runge-Kutta-Nyström method, or a (symplectic) second-order Boris method. In our test runs, we found 
little variation between these two methods - barring numerical instability due to using an insufficiently small step size in time. This is likely because our simulation time is limited by actual computational time, and thus we were unable to realize the slow accumulation of errors in the total energy characteristic of non-symplectic numerical integrators.

With all the particle positions, velocities and accelerations calculated, the numerical radiation spectrum was obtained directly from the Liénard-Wiechert potentials. The radiation spectrum (which is the radiative spectral energy, $d W$ per unit frequency, $\mathrm{d} \omega$ and per unit solid-angle, $\mathrm{d} \eta$ ) seen by a distant observer is given by Landau \& Lifshitz (1975) and Jackson (1999):

$$
\frac{\mathrm{d}^{2} W}{\mathrm{~d} \omega \mathrm{d} \eta}=\frac{e^{2}}{4 \pi^{2} c}\left|\int_{-\infty}^{\infty} \boldsymbol{A}_{\kappa}(t) \mathrm{e}^{\mathrm{i} \omega t} \mathrm{~d} t\right|^{2},
$$

where

$$
\boldsymbol{A}_{\kappa}(t) \equiv \frac{\hat{\boldsymbol{n}} \times[(\hat{\boldsymbol{n}}-\boldsymbol{\beta}) \times \dot{\boldsymbol{\beta}}]}{(1-\hat{\boldsymbol{n}} \cdot \boldsymbol{\beta})^{2}} \mathrm{e}^{-\mathrm{i} \kappa \cdot r(t)} .
$$

In this equation, $\boldsymbol{r}(t)$ is the particle's position at the retarded time $t, \boldsymbol{\kappa} \equiv \hat{\boldsymbol{n}} \omega / c$ is the wave vector which points along $\hat{\boldsymbol{n}}$ from $\boldsymbol{r}(t)$ to the observer and $\dot{\boldsymbol{\beta}} \equiv \mathrm{d} \boldsymbol{\beta} / \mathrm{d} t$. Since the observer is assumed to be distant, $\hat{\boldsymbol{n}}$ is approximated as fixed in time to the origin of the coordinate system.

Next, the total radiation spectrum is obtained by summing over the spectra of the individual particles. For the moment, we will only consider mean free, small-scale magnetic turbulence in the following discussion.

Given an isotropically distributed (in velocity space) ensemble of electrons, the summed spectrum will be equivalent to the angle averaged, i.e. $\mathrm{d} W / \mathrm{d} \omega$, spectrum for a single electron. There are two, usually equivalent, methods for doing this summation. First, one may add the spectra coherently by summing over each particle's $\boldsymbol{A}_{\kappa}$, and then performing a single integration via (3.7). This method is more physical. Alternatively, we may add the spectra incoherently (i.e. by integrating each particle's $\boldsymbol{A}_{\boldsymbol{\kappa}}$ separately, and then summing the results of each integration). As discussed in Hededal (2005), both methods will result in the same spectra since the wave phases are uncorrelated. However, an incoherent sum will produce a spectrum that is less noisy (for a given number of simulation particles) than the coherently summed spectrum. For this reason, we employ the incoherent approach in this study - as we have done previously.

In contrast to our previous studies, (Keenan \& Medvedev 2013; Keenan et al. 2015), the non-vanishing mean magnetic field introduces a previously non-existent anisotropy; the summed spectrum will, as a result, depend upon the location of the observer. However, if the magnetic turbulence is statistically homogeneous/isotropic, then the synchrotron/cyclotron (mean field) component of the spectrum, alone, will possess this dependence. Since the angle-averaged synchrotron spectrum is a known function, we may simply add it to the jitter spectrum, obtained via the summation method above. Lastly, the contribution due to the electric field may be neglected, since $\left\langle E^{2}\right\rangle \ll\left\langle\delta B^{2}\right\rangle$.

Finally, the electron pitch angle (with respect to the $z$-axis) and kinetic energy, $W_{e} \equiv$ $(\gamma-1) m_{e} c^{2}$, were calculated at each time step. Using the definitions, equations (2.3) and (2.14), we obtained the pitch angle and energy diffusion coefficients directly from the simulation data. 


\section{Numerical results}

In $\S 2$, we made a number of theoretical predictions concerning the transport and radiation properties of magnetized plasmas with small-scale turbulent electromagnetic fluctuations - in particular, sub-Larmor-scale Whistler modes. Additionally, we anticipated that an interconnection between the transport and radiative properties of electrons moving through small-scale Whistler turbulence exists, as it does for strictly Weibel-like, mean-field-free turbulence (Keenan \& Medvedev 2013; Keenan et al. 2015).

\subsection{Whistler turbulence}

First of all, we explore the particle transport by testing our predictions concerning the energy and pitch-angle diffusion coefficients in small-scale Whistler turbulence. The diffusion coefficients depend on various parameters, cf. (2.8) and (2.40), namely the particle's velocity, $\beta$, the magnetic fluctuation field strength, $\left\langle\Omega_{\delta B}^{2}\right\rangle$ and the field correlation scale, $\lambda_{B}$.

To start, we must confirm the fundamental assumption of diffusion. As stated previously, a diffusive process requires that both $\left\langle\Delta W_{e}^{2}\right\rangle$ and $\left\langle\alpha^{2}\right\rangle$ increase linearly in time - at least, on some characteristic time scale of the system. With $\delta B / B_{0} \ll 1$, the gyro period

$$
T_{g} \equiv \frac{2 \pi \gamma}{\Omega_{c e}}=2 \pi \frac{\gamma m_{e} c}{e B_{0}},
$$

is such a characteristic, 'macroscopic' time scale. On a multiple gyro period time scale, the electron velocities will change very slightly. Consequently, we may treat the magnitude of the electron velocity as approximately constant.

To establish diffusion, 5000 mono-energetic electrons $(\beta=0.25)$ were injected into Whistler turbulence with $k_{\min }=32 \pi$ (arbitrary simulation units), $k_{\max }=10 k_{\min }$, $\left\langle\delta B^{2}\right\rangle^{1 / 2} / B_{0}=0.1, \Omega_{c e}=1, \rho \approx 400, \chi \approx 0.04$ and $\mu=4$. The simulation time included several gyro periods; $T=10 T_{g}$. Additional simulation parameters include: the time step $\Delta t=0.00125$ (arbitrary units) and the number of Whistler wave modes $N_{m}=10000$. In figure 1 , we see that the average square pitch angle (as measured with respect to the $z$-axis, i.e. the mean field direction) does indeed grow linearly with time. Figure 2 confirms that the electron energy undergoes a classical diffusive process as well. With the existence of pitch angle and energy diffusion established, we then proceeded to compare the slope of $\left\langle\alpha^{2}\right\rangle$ and $\left\langle\Delta W_{e}^{2}\right\rangle$ versus time (the numerical pitch angle and energy diffusion coefficients) to (2.8) and (2.40). In figure 3 , the numerically obtained pitch-angle diffusion coefficients are compared to (2.8) for a range of possible electron velocities. In each, the theoretical and numerical results differ only by a small factor of $O(1)$. Next, in figure 4 , we see decent agreement with (2.40) and the numerical energy diffusion coefficients. Figures 3 and 4, furthermore, confirm that our theoretical diffusion coefficients are valid for all electron velocities - including relativistic speeds.

Another important parameter which strongly influences the diffusive transport is the magnetic field correlation length. In figure 5, the correlation length was varied by changing $k_{\min }$, while keeping all other parameters fixed. Once more, we see close agreement with (2.8). Similarly, the numerical and theoretical energy diffusion coefficients continue to show reasonable agreement - see figure 6 .

We consider the magnetic spectral index, $\mu$ - i.e. the power-law exponent in (2.42). With $k_{\min }=32 \pi$ and $k_{\max }=10 k_{\min }$, we varied the magnetic spectral index, $\mu$ from -3 to 9 . In figure 7 , we see that the numerical pitch-angle diffusion coefficient closely 


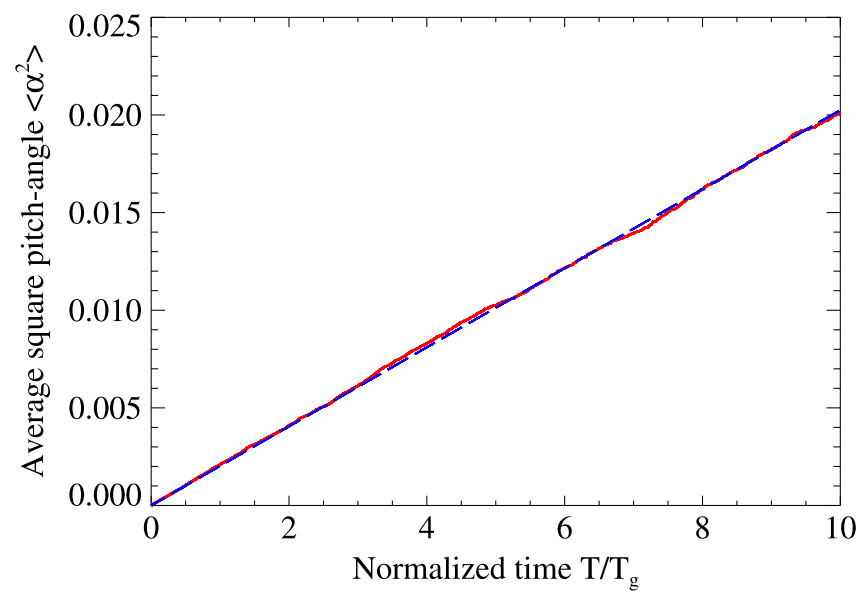

FIGURE 1. Average square pitch angle versus normalized time. Relevant parameters are $\beta=0.25$, (number of simulation particles) $N_{p}=5000, k_{\min }=32 \pi, k_{\max }=10 k_{\text {kmin }}$, $\left\langle\delta B^{2}\right\rangle^{1 / 2} / B_{0}=0.1, \Omega_{c e}=1, \rho \approx 400, \chi \approx 0.04$ and $\mu=4$. The linear nature of the curve (solid, 'red') confirms the diffusive nature of the pitch-angle transport. Here, the dashed ('blue') line indicates a line of best fit (simple linear regression) with Pearson correlation coefficient: 0.9998 .

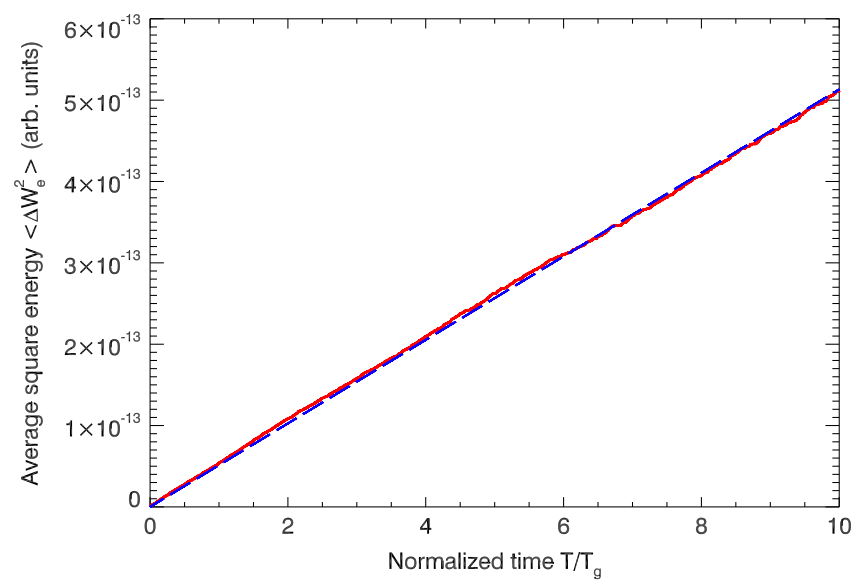

FIGURE 2. Average square change in electron energy (in simulation units) versus normalized time. Relevant parameters are $\beta=0.25$, (number of simulation particles) $N_{p}=$ $5000, k_{\min }=64 \pi, k_{\max }=10 k_{k \min },\left\langle\delta B^{2}\right\rangle^{1 / 2} / B_{0}=0.1, \Omega_{c e}=1, \rho \approx 400, \chi \approx 0.04$ and $\mu=4$. The linear nature of the curve (solid, 'red') confirms the diffusive nature of the energy transport. Here, the dashed ('blue') line indicates a line of best fit (simple linear regression) with Pearson correlation coefficient: 0.9999.

matches the analytical result. Similarly close agreement was, once again, realized between the energy diffusion coefficients; as may be seen in figure 8 .

Finally, we consider the radiation spectra. As discussed in $\S 3$, the radiation spectra are expected to be the summation of synchrotron (cyclotron) and jitter (pseudo-cyclotron) components. For an ultrarelativistic electron, the angle-averaged synchrotron radiation spectrum is the known function (Landau \& Lifshitz 1975; 


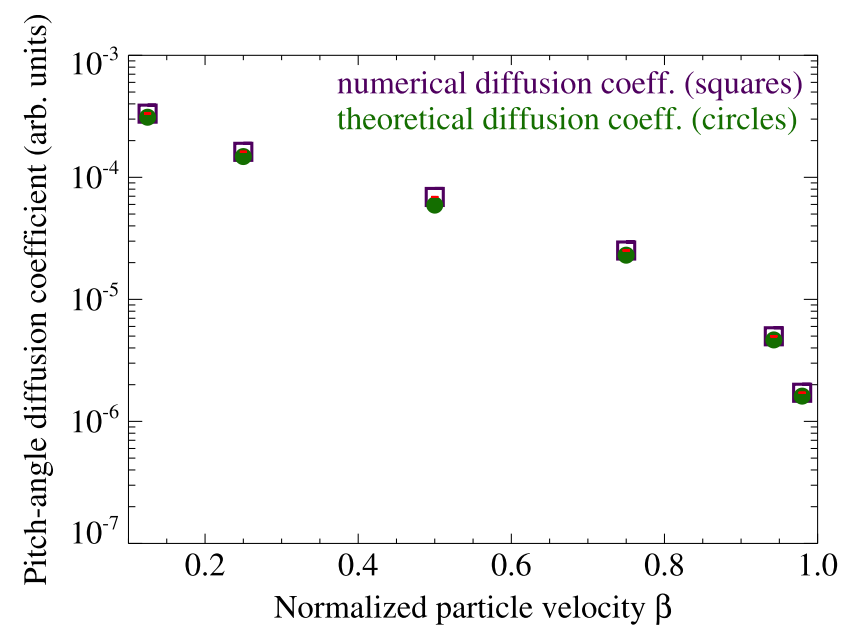

FIGURE 3. Pitch-angle diffusion coefficient, $D_{\alpha \alpha}$ versus the normalized electron velocity, $\beta$. Relevant simulation parameters include: $N_{p}=5000, k_{\min }=32 \pi, k_{\max }=10 k_{\text {min }}$, $\left\langle\delta B^{2}\right\rangle^{1 / 2} / B_{0}=0.1, \Omega_{c e}=1, \chi \approx 0.02$ and $\mu=4$. The (purple) empty 'squares' indicate the $D_{\alpha \alpha}$ 's obtained directly from simulation data (as the slope of $\left\langle\alpha^{2}\right\rangle$ versus time), while the (green) filled 'circles' are the analytical pitch-angle diffusion coefficients, given by (2.8). Numerical error bars (red, lines centred in the 'squares') from the standard deviations on each $\left\langle\alpha^{2}\right\rangle$ also appear. Note: that the symbol sizes are much greater than the statistical/numerical error bars.

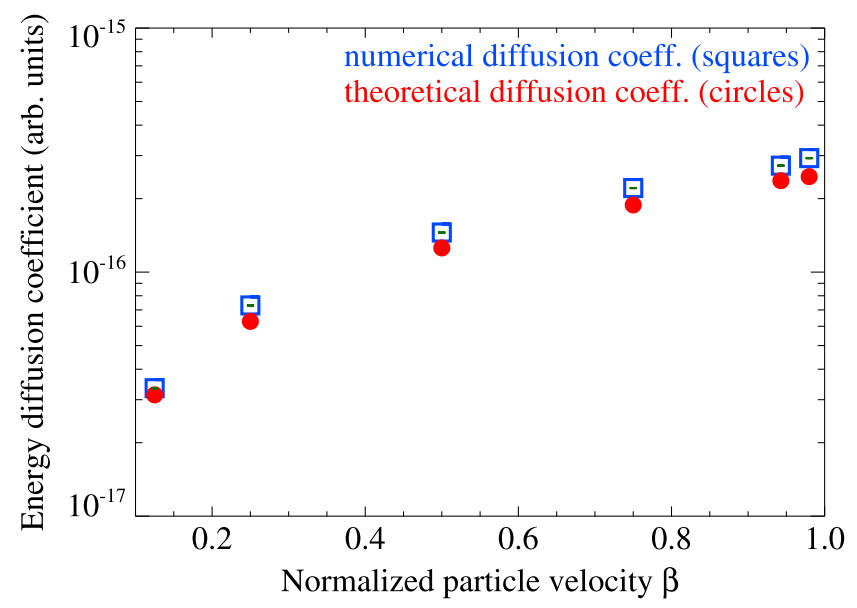

FIGURE 4. Energy diffusion coefficient, $D_{W W}$ versus the normalized electron velocity, $\beta$. Relevant simulation parameters include: $N_{p}=5000, k_{\min }=32 \pi, k_{\max }=10 k_{\text {min }}$, $\left\langle\delta B^{2}\right\rangle^{1 / 2} / B_{0}=0.1, \Omega_{c e}=1, \chi \approx 0.02$ and $\mu=4$. The (blue) empty 'squares' indicate the $D_{W W}$ 's obtained directly from simulation (as the slope of $\left\langle\Delta W_{e}^{2}\right\rangle$ versus time), while the (red) filled 'circles' are the analytical energy diffusion coefficients, given by (2.40). Numerical error bars (green, lines centred in the 'squares') from the standard deviations on each $\left\langle\alpha^{2}\right\rangle$ also appear. Note: that the symbol sizes are much greater than the statistical/numerical error bars. 


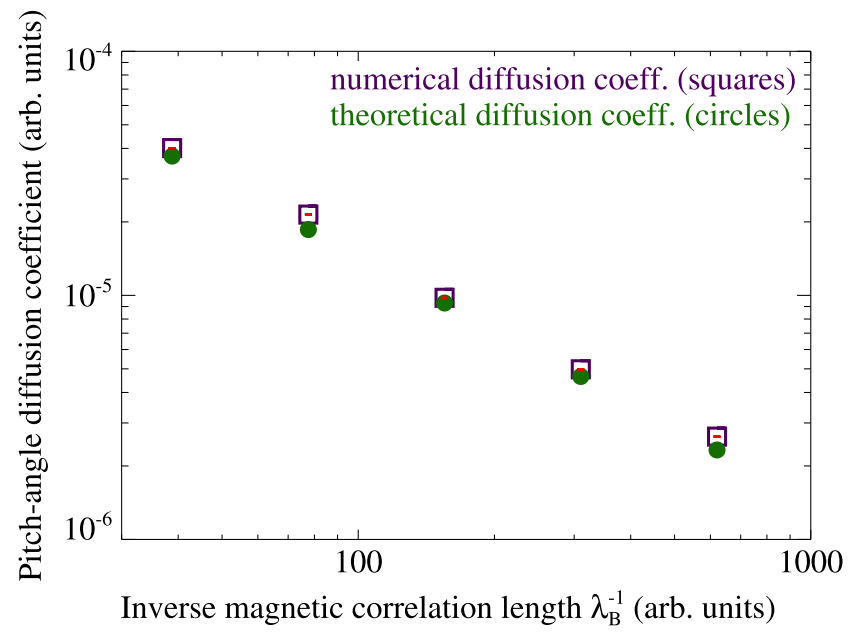

FIGURE 5. Pitch-angle diffusion coefficient, $D_{\alpha \alpha}$ versus the inverse of magnetic field correlation scale, $\lambda_{B}^{-1}$. Relevant simulation parameters include: $\gamma=3, N_{p}=1000, k_{\min }=8 \pi$, $16 \pi, 32 \pi, 64 \pi$ and $128 \pi, k_{\max }=10 k_{k \min }$ (for each $k_{k \min }$ ), $\left\langle\delta B^{2}\right\rangle^{1 / 2} / B_{0}=0.1, \Omega_{c e}=1$, $\chi \approx 0.02$ and $\mu=4$. For each data point, the theoretical and numerical results differ only by a small factor of $O(1)$.

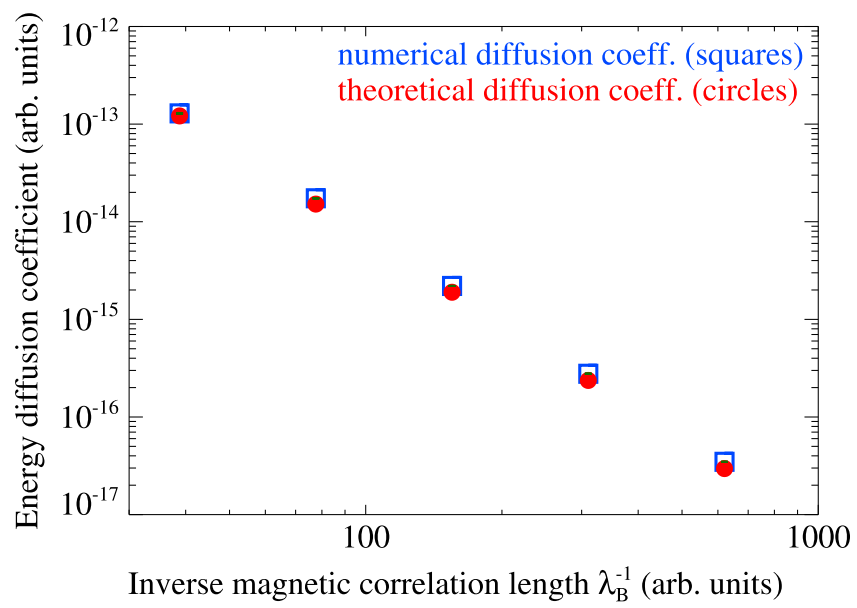

FIGURE 6. Energy diffusion coefficient, $D_{W W}$ versus the inverse of magnetic field correlation scale, $\lambda_{B}^{-1}$. Relevant simulation parameters include: $\gamma=3, N_{p}=1000, k_{\min }=8 \pi$, $16 \pi, 32 \pi, 64 \pi$ and $128 \pi, k_{\max }=10 k_{k \min }$ (for each $k_{k \min }$ ), $\left\langle\delta B^{2}\right\rangle^{1 / 2} / B_{0}=0.1, \Omega_{c e}=1$, $\chi \approx 0.02$ and $\mu=4$. The theoretical and numerical results differ only by a small factor of $O(1)$.

Jackson 1999):

$$
\frac{\mathrm{d} W}{\mathrm{~d} \omega}=\sqrt{3} \frac{e^{2}}{c} \gamma \frac{\omega}{\omega_{c}} \int_{\omega / \omega_{c}}^{\infty} \mathrm{K}_{5 / 3}(x) \mathrm{d} x,
$$

where $\mathrm{K}_{j}(x)$ is a modified Bessel function of the second kind, and $\omega_{c}=3 / 2 \gamma^{2} \Omega_{c e}$ is the critical synchrotron frequency. This result applies for an electron moving in the 


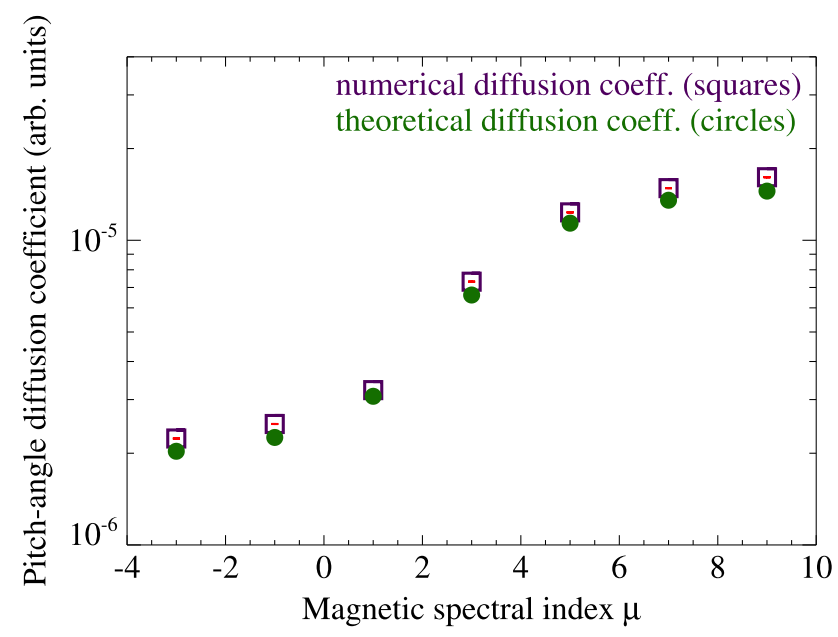

FIGURE 7. Pitch-angle diffusion coefficient, $D_{\alpha \alpha}$ versus the magnetic spectral index, $\mu$. Relevant parameters are $N_{p}=2000, k_{\min }=32 \pi, k_{\max }=10 k_{\max },\left\langle\delta B^{2}\right\rangle^{1 / 2} / B_{0}=0.1$, $\Omega_{c e}=1$ and $\chi \approx 0.05$. Notice that the numerical results have nearly the same functional dependence on $\mu$ as the analytical squares, as given by (2.8).

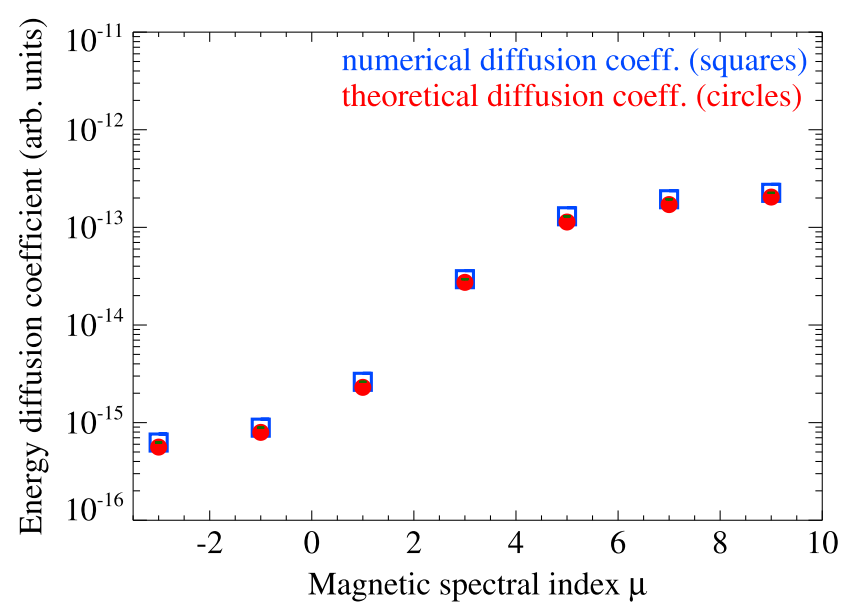

FIGURE 8. Energy diffusion coefficient, $D_{W W}$ versus the magnetic spectral index, $\mu$. Relevant parameters are $N_{p}=2000, k_{\min }=32 \pi, k_{\max }=10 k_{\max },\left\langle\delta B^{2}\right\rangle^{1 / 2} / B_{0}=0.1, \Omega_{c e}=1$ and $\chi \approx 0.05$.

plane transverse to the ambient magnetic field, i.e. when $\alpha=\pi / 2$. Nonetheless, we find the expression fits the synchrotron components fairly well; especially when $\gamma$ is reasonably large.

We illustrate two numerical spectra here, along with their corresponding analytical estimates - for details concerning the jitter component, see Keenan et al. (2015). First, we considered a $\gamma=5$ electron population for figure 9. In this plot, the relevant parameters are: $N_{p}=1000, \Delta t=0.00125, k_{\min }=2 \pi, k_{\max }=20 \pi,\left\langle\delta B^{2}\right\rangle^{1 / 2} / B_{0}=0.1$, $\Omega_{c e}=0.512, \mu=4, \rho \approx 928, \chi \sim 1$ and the total simulation time was $T=5 T_{g}$. We see that the synchrotron + jitter fit closely resembles the numerical spectrum. 


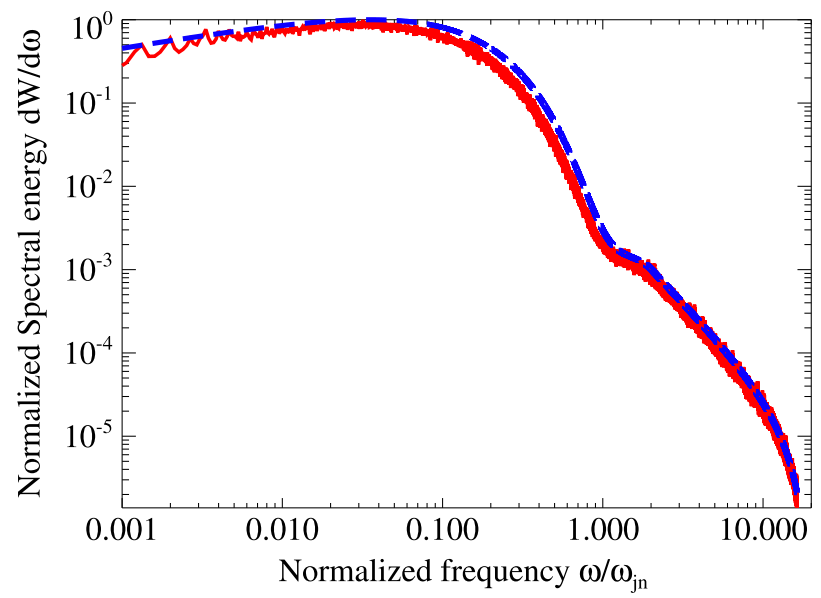

FIGURE 9. Radiation spectrum for a mono-energetic, isotropic distribution of $\gamma=5$ $\left(\chi \sim 1 ; \rho \approx 928 ;\left\langle\delta B^{2}\right\rangle^{1 / 2} / B_{0}=0.1\right)$ electrons moving through small-scale Whistler turbulence. The frequency is normalized by $\omega_{j n}=\gamma^{2} k_{\min } \beta c-$ the relativistic jitter frequency. The solid ('red') curve is from simulation data, whereas the dashed ('blue') curve is the analytic estimate. Clearly, the spectrum is well represented by a superposition of synchrotron + jitter components. Note the lower-frequency synchrotron component and a higher-frequency power-law component corresponding to the small-angle jitter radiation.

Next, we explored the non-relativistic regime. In figure 10, we assumed a population of sub-Larmor-scale $\beta=0.125$ electrons. As expected, a peak in the spectrum may be observed near the cyclotron frequency $\Omega_{c e}$ - confirming that the total spectrum is the hybrid of pseudo-cyclotron + cyclotron radiation. Additionally, to provide a point of comparison, we have superimposed a simulation result for $\gamma=4$ electrons.

\section{Conclusions}

In this paper we explored test particle transport (diffusion) and radiation production in magnetized plasmas with small-scale electromagnetic turbulence. In our previous works (Keenan \& Medvedev 2013; Keenan et al. 2015), we showed that the pitchangle diffusion coefficient and the simultaneously produced radiation spectrum are wholly determined by the particle velocity and the statistical/spectral properties of small-scale (mean free) magnetic turbulence. Here, we have generalized these results to the case when the magnetic field has a mean value.

In fact, the pitch-angle diffusion coefficient, equation (2.8), remains unchanged by the addition of a mean field - so long as the pitch angle, $\alpha$, assumes its conventional meaning, i.e. as the angle between the electron velocity vector and the ambient (mean) magnetic field. Since magnetized plasmas characterized by instability often include random electric fields, as is the case for the Whistler turbulence considered here, we additionally considered test particle energy diffusion. We showed that the energy diffusion coefficient in small-scale Whistler turbulence is directly proportional to the (magnetic) pitch-angle diffusion coefficient - see (2.40). Thus, it is also intimately related to the field's statistical properties. Consequently, transport via energy diffusion may provide yet another powerful diagnostic tool for the investigation of small-scale electromagnetic fluctuations in magnetized plasmas. 


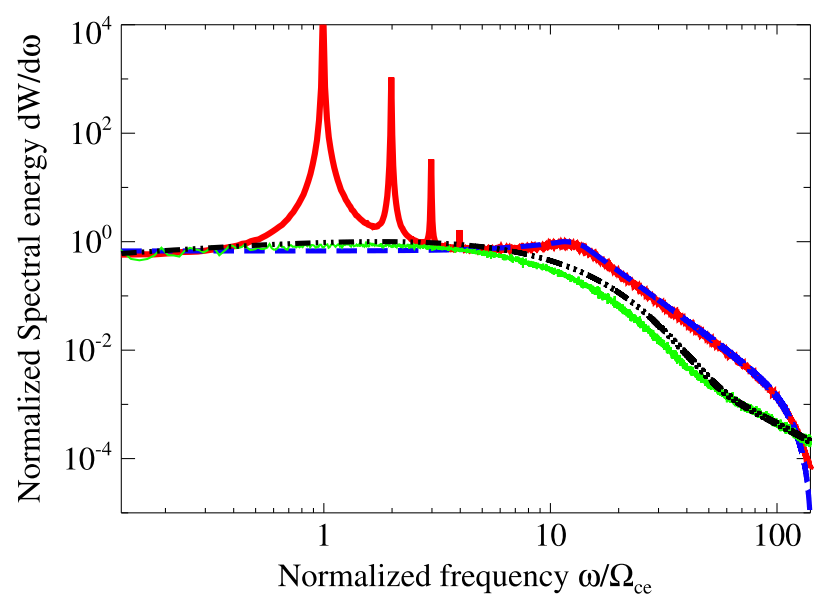

FIGURE 10. Radiation spectrum for a mono-energetic, isotropic distribution of $\beta=0.125$ electrons $\left(\chi \sim 0.04 ; \rho \approx 160 ;\left\langle\delta B^{2}\right\rangle^{1 / 2} / B_{0}=0.2 ; \Omega_{c e}=2 ; k_{\min }=64 \pi ; k_{\max }=10 k_{\min }\right.$; $\mu=5 ; T=50 T_{g}$ ); superimposed with a spectrum given a population of $\gamma=4$ electrons $\left(\chi \sim 1 ; \rho \approx 367 ;\left\langle\delta B^{2}\right\rangle^{1 / 2} / B_{0}=0.1 ; \Omega_{c e}=0.512 ; k_{\min }=\pi ; k_{\max }=10 \pi ; \mu=4 ; T=5 T_{g}\right)$. The normalization on the $y$-axis is arbitrary, whereas the $x$-axis is normalized to the $\beta=0.125$ population's cyclotron frequency, i.e. $\Omega_{c e}=2$. The 'thick' solid ('red') curve is from simulation data for the $\beta=0.125$ population, the dashed ('blue') curve is the corresponding analytic estimate for pure pseudo-cyclotron radiation, the 'thin' solid line is the simulation data for the $\gamma=4$ population and the 'dot-dashed' ('black') line is the $\gamma=4$ analytic estimate. Notice, for the $\beta=0.125$ spectrum, that the spectrum peaks near the cyclotron frequency, $\Omega_{c e}$ - hence we see the signature of cyclotron radiation. The additional harmonics, which are purely a relativistic effect, are the signature of emerging synchrotron radiation.

Finally, we confirmed that the test particle radiation spectrum (which is predominately determined by the magnetic field in Whistler turbulence) is simply the summation of a small-scale, jitter/pseudo-cyclotron component and a regular, synchrotron/cyclotron component - see (2.49). We have, further, confirmed these theoretical results via first-principle numerical simulations.

Our model implicitly considered a scenario whereby a turbulent magnetic field was generated in a cold, magnetized, background plasma. We then imagined the existence of a hot population of sub-Larmor-scale electrons that served as our test particles. We suggested that the motion of high energy, supra-thermal, super-halo electrons through the magnetized solar wind is a promising candidate for the physical realization of our model. Indeed, despite the fact that this population only accounts for a small fraction of a percent of the solar wind, its high energy (2-20 keV) makes it very significant (Wang et al. 2015; Yoon 2015).

Additionally, the super-halo population is largely insensitive to solar activity, and it is likely constantly present in the interplanetary plasma (Wang et al. 2015) - thus, it is a relatively fixed source of high-energy particles. In fact, recent work has suggested that the super-halo electrons may mediate Weibel-like instabilities in the solar wind plasma - facilitating the development of kinetic-Alfvén wave (KAW) and/or Whistlermode turbulence at sub-electron spatial scales (Che et al. 2014).

The nature of this wave turbulence in the solar wind plasma is a matter of contention. Conflicting accounts implicate either KAW or Whistler modes (or both) 
(Mithaiwala et al. 2012; Salem et al. 2012). A number of reasons for this ambiguity have been given. For example, in situ measurements of these waves must be done in the spacecraft frame - which is usually moving at super-Alfvénic speeds with respect to the plasma (Salem et al. 2012). Furthermore, the solar wind hosts a permanent source of turbulence; hence, many results implicating Whistler waves - via, for example, the observed power spectrum - may be the erroneous signature of the ever present background turbulence (Lacombe et al. 2014).

However, a more detailed analysis of the turbulent spectrum may provide a means by which Whistlers and KAW may be distinguished. In fact, the degree of anisotropy has been found to significantly differ between the two types of wave turbulence (Salem et al. 2012). With regard to our model, the presence of anisotropy will result in diffusion coefficients that differ perpendicular and parallel to the anisotropy axis (which is typically the direction of the ambient magnetic field), since the correlation lengths will depend upon the structure of the correlation tensor.

Hence, we may imagine that the transport properties of hot electrons (e.g. sub-Larmor scale, super-halo electrons) may be different for Whistler-mode and KAW turbulence. The radiation spectrum would, additionally, distinguish these forms of turbulence - as the anisotropy, which features into the field correlation tensor, would alter the shape of the radiation spectrum in a characteristic way.

Concerning our energy diffusion coefficient, our model's principal limitation is the essential assumption of the cold plasma approximation. In many cases, thermal effects must be accounted for; i.e. the plasma beta is non-negligible. Nonetheless, under certain conditions, the underlying plasma may be considered cold. As an example, the plasma outflow in ultrarelativistic collisionless shocks is beam-like, with very little dispersion; this permits a cold plasma treatment (Lemoine \& Pelletier 2010). Therefore, since these shocks may be mediated in part by small-scale Whistler modes, our rough estimates concerning the diffusive transport of electrons may provide some insight into the process of shock acceleration.

To conclude, the obtained results reveal strong inter-relation of transport and radiative properties of plasmas turbulent at sub-Larmor scales - magnetized, i.e. possessing a mean magnetic field, or otherwise.

\section{Acknowledgements}

This work was partially supported by the DOE grant DE-FG02-07ER54940 and the NSF grant AST-1209665.

\section{REFERENCES}

Bai, X.-N., Caprioli, D., Sironi, L. \& Spitkovsky, A. 2015 Simulations of ion acceleration at non-relativistic shocks. II. Magnetic field amplification. Astrophys. J. 809, 55.

Bell, A. R. 1978 The acceleration of cosmic rays in shock fronts - I. Mon. Not. R. Astron. Soc. 182, 147.

BELL, A. R. 2004 Turbulent amplification of magnetic field and diffusive shock acceleration of cosmic rays. Mon. Not. R. Astron. Soc. 353, 550.

Bell, A. R. 2005 The interaction of cosmic rays and magnetized plasma. Mon. Not. R. Astron. Soc. 358, 181.

Brambilla, M. 1998 Kinetic Theory of Plasma Waves: Homogeneous Plasmas, International Series of Monographs on Physics, vol. 96. Clarendon.

Bykov, A. M., Brandenburg, A., Malkov, M. A. \& Osipov, S. M. 2013 Microphysics of cosmic ray driven plasma instabilities. Space Sci. Rev. 178, 201. 
CAPRIOli, D. \& SpItKovsky, A. 2014 Simulations of ion acceleration at non-relativistic shocks. II. Magnetic field amplification. Astrophys. J. 794, 46.

Chang, S. S., Ni, B. B., Bortnik, J., Zhou, C., Zhao, Z. Y., Li, J. X. \& Gu, X. D. 2014 Resonant scattering of energetic electrons in the plasmasphere by monotonic whistler-mode waves artificially generated by ionospheric modification. Ann. Geophys. 32, 507.

Che, H., Goldstein, M. L. \& ViÑAs, A. F. 2014 Bidirectional energy cascades and the origin of kinetic Alfvénic and whistler turbulence in the solar wind. Phys. Rev. Lett. 112, 061101.

Chen, F. F. 1984 Introduction to Plasma Physics and Controlled Fusion, vol. 1, 2nd edn. Plenum.

FAIRFIELD, D. H. 1974 Whistler waves observed upstream from collisionless shocks. J. Geophys. Res. 79, 1368.

Frederiksen, J. T., Hededal, C. B., Haugbølle, T. \& Nordlund, A 2004 Magnetic field generation in collisionless shocks: pattern growth and transport. Astrophys. J. Lett. 608, L13.

Fried, B. D. 1959 Mechanism for instability of transverse plasma waves. Phys. Fluids 2, 337.

GARY, S. P. \& KARIMABADI, H. 2006 Linear theory of electron temperature anisotropy instabilities: whistler, mirror, and Weibel. J. Geophys. Res. 111, A11224.

Giacalone, J. \& JoKipII, J. R. 1999 The transport of cosmic rays across a turbulent magnetic field. Astrophys. J. 520, 204.

HededAL, C. 2005 Gamma-ray bursts, collisionless shocks and synthetic specta. PhD thesis, Niels Bohr Institute in Denmark.

Huntington, C. M., Fiuza, F., Ross, J. S., Zylstra, A. B., Drake, R. P., Froula, D. H., Gregori, G., Kugland, N. L., Kuranz, C. C., Levy, M. C. et al. 2015 Observation of magnetic field generation via the Weibel instability in interpenetrating plasma flows. Nat. Phys. 11 (2), 173-176.

JACKSON, J. D. 1999 Classical Electrodynamics, 3rd edn. Wiley.

Keenan, B. D., Ford, A. L. \& Medvedev, M. V. 2015 Transport of and radiation production by transrelativistic and nonrelativistic particles moving through sub-Larmor-scale electromagnetic turbulence. Phys. Rev. E 92, 033104.

Keenan, B. D. \& Medvedev, M. V. 2013 Particle transport and radiation production in sub-Larmorscale electromagnetic turbulence. Phys. Rev. E 88, 013103.

Lacombe, C., Alexandrova, O., Matteini, L. et al. 2014 Whistler mode waves and the electron heat flux in the solar wind: cluster observations. Astrophys. J. 796, 5.

Landau, L. D. \& Lifshitz, E. M. 1975 The Classical Theory of Fields, Course of Theoretical Physics, vol. 2. Pergamon.

Lazar, M., Poedts, S., Schlickeiser, R. \& Ibscher, D. 2014 The electron firehose and ordinarymode instabilities in space plasmas. Solar Phys. 289, 369.

Lemoine, M. \& Pelletier, G. 2010 On electromagnetic instabilities at ultra-relativistic shock waves. Mon. Not. R. Astron. Soc. 402, 321.

Lengyel-Frey, D., Hess, R. A., MacDowall, R. J. et al. 1996 Ulysses observations of whistler waves at interplanetary shocks and in the solar wind. J. Geophys. Res. 101, 27555.

LIN, R. P. 1998 WIND observations of suprathermal electrons in the interplanetary medium. Space Sci. Rev. 86, 61 .

Lin, N., KellogG, P. J., MacDowall, R. J. et al. 1998 Very low frequency waves in the heliosphere: ulysses observations. J. Geophys. Res. 103, 12023.

LuceK, S. G. \& Bell, A. R. 2000 Non-linear amplification of a magnetic field driven by cosmic ray streaming. Mon. Not. R. Astron. Soc. 314, 65.

MedvedeV, M. V. 2000 Theory of 'Jitter' radiation from small-scale random magnetic fields and prompt emission from gamma-ray burst shocks. Astrophys. J. 40, 704.

Medvedev, M. V. 2006 The theory of spectral evolution of the gamma-ray burst prompt emission. Astrophys. J. 637, 869.

Medvedev, M. V. 2009a Physics of Relativistic Shocks, American Institute of Physics Conference Series, vol. 1183, p. 189. American Institute of Physics.

MedvedeV, M. V. 2009 b Radiation of electrons in Weibel-generated fields: a general case. Astrophys. Space Sci. 322, 147. 
Medvedev, M. V., Frederiksen, J. T., Haugbølle, T. \& Nordlund, A 2011 Radiation signatures of sub-larmor scale magnetic fields. Astrophys. J. 737, 55.

Mithaimala, M., Rudakov, L., Crabtree, C. \& Ganguli, G. 2012 Co-existence of whistler waves with kinetic Alfven wave turbulence for the high-beta solar wind plasma. Phys. Plasmas 19, 102902.

Mondal, S., NARAYAnan, V., Ding, W. J. et al. 2012 Direct observation of turbulent magnetic fields in hot, dense laser produced plasmas. Proc. Natl Acad. Sci. USA 109, 8011.

Nishikawa, K.-I., Hardee, P., Richardson, G. et al. 2003 Particle acceleration in relativistic jets due to Weibel instability. Astrophys. J. 595, 555.

Palodhi, L., Califano, F. \& Pegoraro, F. 2010 On the transition between the Weibel and the Whistler instabilities. Plasma Phys. Control. Fusion 52, 095007.

Ramírez Vélez, J. C., Blanco-Cano, X., Aguilar-Rodriguez, E. et al. 2012 Whistler waves associated with weak interplanetary shocks. J. Geophys. Res. 117, A11103.

Ren, C., Tzoufras, M., Tsung, F. S. et al. 2004 Global simulation for laser-driven MeV electrons in fast ignition. Phys. Rev. Lett. 93, 185004.

Reville, B. \& KiRK, J. G. 2010 Computation of synthetic spectra from simulations of relativistic shocks. Astrophys. J. 724, 1283.

Salem, C. S., Howes, G. G., SundKvist, D. et al. 2012 Identification of kinetic Alfven wave turbulence in the solar wind. Astrophys. J. Lett. 745, L9.

SAZHIn, S.-S. 1993 Whistler-Mode Waves in a Hot Plasma, Cambridge Atmospheric and Space Science Series. Cambridge University Press.

Schaefer-Rolffs, U., Lerche, I. \& TAUtz, R. C. 2009 Particle radiation from relativistic plasmas contained by soliton waves. J. Phys. A: Math. Theoret. 42, 105501.

SCHLICKeISER, R. 1994 Cosmic-ray transport and acceleration. Astrophys. J. Suppl. 90, 929.

Sironi, L. \& SPITKOVSKY, A. 2011 Particle acceleration in relativistic magnetized collisionless electron-ion shocks. Astrophys. J. 726, 75.

SiRONI, L. \& SPITKOVSKY, A. 2014 Relativistic reconnection: an efficient source of non-thermal particles. Astrophys. J. Lett. 783, L21.

Sironi, L., Spitkovsky, A. \& ARONS, J. 2013 The maximum energy of accelerated particles in relativistic collisionless shocks. Astrophys. J. 771, 54.

SPITKovsky, A. 2008 On the structure of relativistic collisionless shocks in electron-ion plasmas. Astrophys. J. Lett. 673, L39.

Stix, T. H. 1992 Waves in Plasmas. (ed. H. Thomas), American Institute of Physics.

TAutz, R. C. \& Lerche, I. 2012 Radiation from non-linear Weibel plasma modes. Phys. Rep. 520, 1.

Tatarakis, M., Beg, F. N., Clark, E. L. et al. 2003 Propagation instabilities of high-intensity laser-produced electron beams. Phys. Rev. Lett. 90, 175001.

TAutz, R. C. \& Dosch, A. 2013 On numerical turbulence generation for test-particle simulations. Phys. Plasmas 20, 022302.

Teraki, Y. \& TAKahara, F. 2011 A novel emission spectrum from a relativistic electron moving in a random magnetic field. Astrophys. J. 735, L44.

Toptygin, I. N. \& Fleishman, G. D. 1987 A role of cosmic rays in generation of radio and optical radiation by plasma mechanisms. Astrophys. Space Sci. 132, 213.

Tsurutani, B. T., Smith, E. J. \& Jones, D. E. 1983 Waves observed upstream of interplanetary shocks. J. Geophys. Res. 88, 5645.

Verkhoglyadova, O. P., Tsurutani, B. T. \& Lakhina, G. S. 2010 Properties of obliquely propagating chorus. J. Geophys. Res. 115, A00F19.

WANG, L., YANG, L., HE, J. et al. 2015 Solar wind $\sim 20-200 \mathrm{keV}$ superhalo electrons at quiet times. Astrophys. J. Lett. 803, L2.

WeIBEL, E. S. 1959 Spontaneously growing transverse waves in a plasma due to an anisotropic velocity distribution. Phys. Rev. Lett. 2, 83.

Yoon, P. H. 2015 Solar wind electron energization by plasma turbulence. J. Phys.: Conf. Ser. 642, 012030 .

Zheleznyakov, V. V. \& Trakhtengerts, V. Y. 1966 Influence of the medium on generation of type-IV solar radio emission. Sov. Astron. 9, 775. 